\title{
Micobacterias atípicas en cinco pacientes adultos sin evidencias de inmunosupresión. Construyendo una experiencia
}

\author{
Alberto Fica, Andrés Soto, Jeannette Dabanch, Lorena Porte, \\ Marcelo Castro, Luis Thompson y M. Elvira Balcells
}

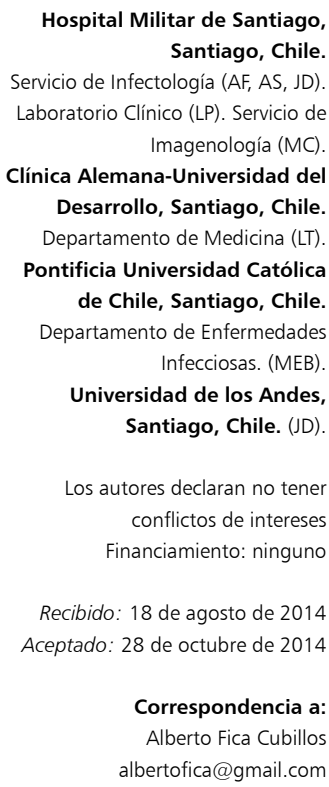

Hospital Militar de Santiago, Santiago, Chile. Servicio de Infectología (AF, AS, JD) Laboratorio Clínico (LP). Servicio de Imagenología (MC) Clínica Alemana-Universidad de Desarrollo, Santiago, Chile. Departamento de Medicina (LT) Pontificia Universidad Católica de Chile, Santiago, Chile. Departamento de Enfermedades Infecciosas. (MEB) Universidad de los Andes, Santiago, Chile. (JD).

Los autores declaran no tener conflictos de intereses Financiamiento: ninguno

Recibido: 18 de agosto de 2014 Aceptado: 28 de octubre de 2014

Correspondencia a: Alberto Fica Cubillos albertofica@gmail.com

\section{Atypical mycobacterial infections in five adult patients without evidence of immunosuppression. Making an experience}

We aim to communicate the experience gathered during the management of infections by atypical mycobacteria in immunocompetent patients in a general practice. Between 2008 and 2013, 5 patients with non-tuberculous mycobacterial infections were identified: 2 with cutaneous involvement and 3 with lung infection. None of them had evidence of immunosuppression. A patient with elbow bursitis by $M$. chelonae presented with a high mononuclear count in fluid analysis with mycobacterial growth at the fifth day of culture. He evolved satisfactorily with clarithromycin. A case with $M$. fortuitum skin infection had a delayed initial diagnosis with progression to local draining lymph nodes; the culture when requested was positive after 13 days of incubation. Patients with pulmonary infection presented with prolonged cough and sputum and had in common to be postmenopausal women displaying small nodules and bronchiectases at lung images, a classical pattern. Time elapsed between respiratory sampling and a definitive inform ranged from 40 to 89 days. Non-tuberculous mycobacterial infections in non-immunosuppresed patients can generate diagnostic and therapeutic challenges. Delay in identification contributes to this problem.

Key words: Atypical mycobacteria, Mycobacterium intracellulare, Mycobacterium kansasii, Mycobacterium chelonae, Mycobacterium fortuitum, lung diseases, bursitis, HIV, tuberculosis, therapeutics, treatment outcome.

Palabras clave: Micobacteria atípica, Mycobacterium intracellulare, Mycobacterium kansasii, Mycobacterium chelonae, Mycobacterium fortuitum, enfermedad pulmonar, bursitis, VIH, tuberculosis, tratamiento, desenlace.

\section{Introducción}

L as micobacterias atípicas, ambientales o no tuberculosas, comprenden un amplio grupo de especies que supera el centenar y que adquirieron notoriedad clínica por la pandemia de SIDA. Se encuentran en forma ubicua en el suelo o agua, no se les considera transmisible entre humanos y tampoco, hasta donde se sabe, constituyen una zoonosis. A diferencia de la tuberculosis (TBC), no establecen una infección latente con reactivación posterior. Su identificación se ha facilitado por la modernización de los laboratorios de microbiología que han incorporado técnicas moleculares. Varios estudios dan cuenta de un aumento de los aislamientos clínicos de este grupo de agentes, especialmente entre la población sin infección por VIH o SIDA en países desarrollados. Por ejemplo, en el Reino Unido se ha observado un incremento en el número de aislamientos desde 0,9 por 100.000 el año 1995 a cifras de 2,9 casos por 100.000 el $2006^{1,2}$. Este aumento de casos se ha focalizado especialmente en personas a partir de los 65 años e incluye varias especies, especialmente en muestras de origen pulmonar'. La relevancia de este problema emergente parece también estar ocurriendo en Chile ya que se ha observado un incremento en el porcentaje de aislamiento de este tipo de micobacterias desde 2,8\% reportado por el Instituto de Salud Pública de Chile (ISP) para el año 1970 a $8,3 \%$ en el $2008^{3}$. Este fenómeno no es explicado por una expansión en los pacientes con SIDA ya que, por ejemplo, sólo 6 de 48 cepas de este grupo identificadas por el ISP el 2008 estaban asociadas a esta condición ${ }^{3}$. La experiencia publicada sobre estas micobacterias es muy reducida en Chile, quedando restringida a unos pocos casos descritos en pacientes con inmunodeficiencias congénitas ${ }^{4} \mathrm{o}$ adquiridas como en VIH/SIDA ${ }^{5,6}$, a un brote en pacientes inmunocompetentes afectados por extractos naturales aplicados en piel con interés cosmético ${ }^{7} \mathrm{y}$ a dos casos de infección pulmonar por Mycobacterium avium complex en pacientes inmunocompetentes ${ }^{8}$.

Es objetivo de esta casuística comunicar la experiencia acumulada en cinco pacientes con infecciones por micobacterias atípicas pulmonares o extra-pulmonares y que no tenían evidencias de inmunosupresión.

\section{Pacientes y Métodos}

Los pacientes fueron identificados desde el registro del Laboratorio Clínico del Hospital Militar de Santiago que 
mantiene la información sobre la identificación de micobacterias confirmadas por el ISP. Se incluye también un caso detectado en Clínica Alemana de Santiago, manejado en forma conjunta con el Hospital Clínico de la Pontificia Universidad Católica de Chile. La información clínica, de laboratorio y evolución se presenta en tablas sumatorias para facilitar la lectura de los rasgos más importantes. No se aplicaron pruebas estadísticas por el reducido número de los casos. El período de análisis abarca desde el 2008 al 2013.

Este estudio fue aprobado por el Comité de Ética del Hospital Militar.

\section{Resultados}

Desde el año 2008 hasta fines de 2013 se reportan cinco pacientes con infección por micobacterias atípicas, tres de ellos con muestras respiratorias, uno con una muestra de líquido de bursa del codo izquierdo y uno con cultivo de secreción cutánea (Tabla 1). Todos los pacientes se atendieron en forma ambulatoria a excepción del caso de infección cutánea profunda que requirió de escisión quirúrgica.

\section{Bursitis de codo}

Se trató de un varón de 54 años atendido el año 2008 (caso 1) por una bursitis de codo (Figura 1) con tres semanas de evolución. La punción de la bursa reveló un líquido hemorrágico con 1.500 leucocitos $/ \mathrm{mm}^{3}$ con $90 \%$ de tipo mononuclear y glucosa de $89 \mathrm{mg} / \mathrm{dL}$; la tinción Gram no reveló bacterias. La eritrosedimentación era normal, el paciente no tenía anemia y el test ELISA para VIH fue negativo. El cultivo en medios habituales (agar sangre) demostró al quinto día, el desarrollo de colonias de color blanco con aspecto de tiza húmeda y la tinción de Ziehl-Neelsen demostró una bacteria filamentosa ácidoalcohol resistente. La muestra fue derivada al Instituto de Salud Pública (ISP) y el informe final indicó la presencia de Mycobacterium chelonae. El paciente fue tratado con claritromicina por seis meses y tuvo una resolución completa del cuadro sin secuelas.

\section{Nódulo cutáneo ulcerado con adenitis regional supurada}

Correspondió a una mujer de 42 años, previamente sana, que consultó en agosto de 2013 por aparición de una lesión ulcerada en la pantorrilla derecha. No refería heridas o puertas de entradas evidentes en la historia previa, salvo depilación habitual con cera. Recibió un curso de antibioterapia empírica sin mejoría (amoxicilina/ác. clavulánico), profundizándose la lesión de tejidos blandos y aparición de zona necrótica en la piel. Los exámenes generales estaban en rango normal y una prueba de ELISA para VIH negativa. En octubre de 2013 se resecó

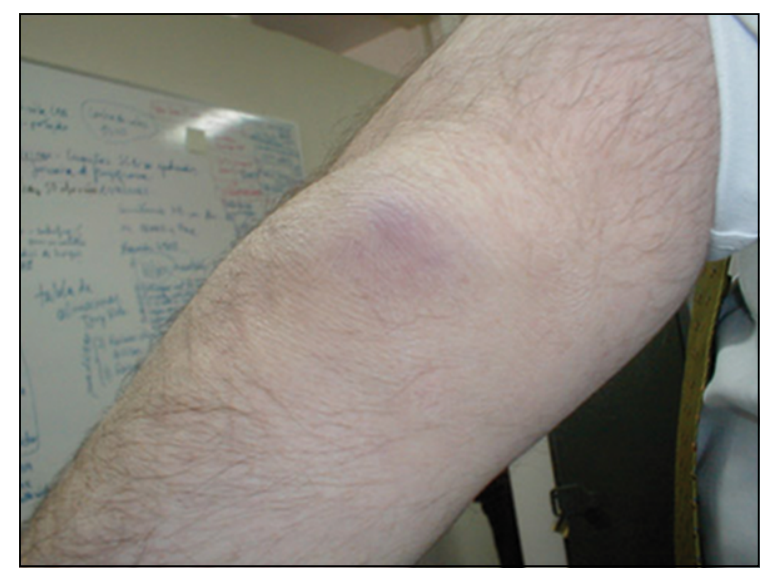

Figura 1. Imagen de bursitis del codo de uno de los casos.

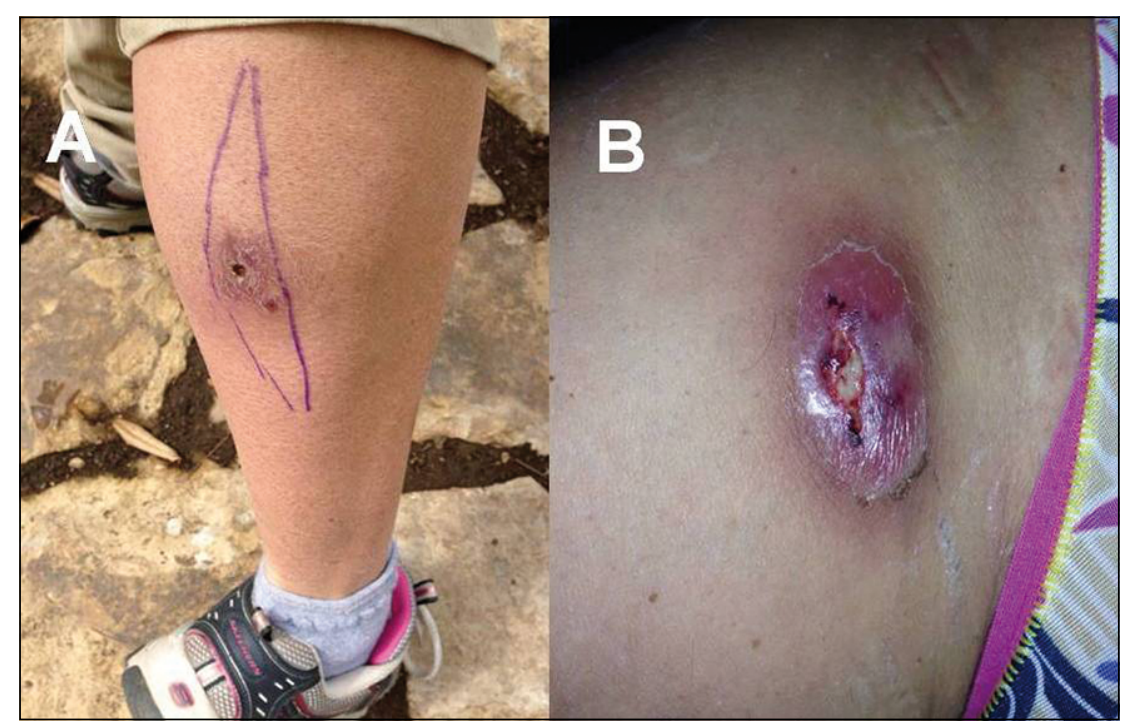

Figura 2. A) Lesión ulcerada con infiltración de tejidos blandos profundos y tendencia a la necrosis central, en la pantorilla derecha. B) Adenopatía inguinal derecha fistulizada a la piel (el mismo paciente).

la lesión ulcerada, pero a las dos a tres semanas recidivó el aumento de volumen de la pantorrilla y aparecieron grandes adenopatías inguinales derechas con supuración y necrosis de la piel (Figura 2). La biopsia fue informada como "granulomas con presencia de bacilos ácido-alcohol resistentes". La TAC de tórax fue normal y la ecotomografía de la pierna y zona inguinal derecha reveló un marcado engrosamiento del tejido celular subcutáneo, con un trayecto fistuloso, de aproximadamente $6,5 \mathrm{~mm}$ de longitud, y 2,3 $\mathrm{mm}$ de espesor. En la región inguinal derecha se demostraron múltiples adenopatías reactivas, hipervascularizadas al Doppler, la mayor de las cuales medía $2 \mathrm{~cm}$. A comienzos de 2013 se inició tratamiento anti tuberculoso empírico con obtención de cultivo para micobacterias de la secreción del ganglio inguinal, la 


\begin{tabular}{|c|c|c|c|c|c|}
\hline & Caso 1 & Caso 2 & Caso 3 & Caso 4 & Caso 5 \\
\hline Año de consulta & 2008 & 2013 & 2011 & 2012 & 2010 \\
\hline Edad en años/sexo & 54/masculino & 42/femenino & 78/femenino & 59/femenino & 87/femenino \\
\hline Co-morbilidad & & No & EPOC & Asma & No \\
\hline Tabaco/alcohol & & No & No/No & $\mathrm{No} / \mathrm{No}$ & No/No \\
\hline Antecedentes TBC & & No & Esofagitis TBC a los 56 años & No & No \\
\hline Corticosteroides sistémicos & No & No & No & No & No \\
\hline Síntoma principal & Bursitis de codo & $\begin{array}{l}\text { Nódulo ulcerado de la } \\
\text { pantorrilla }\end{array}$ & Baja de peso $10 \%$ & Tos con expectoración & Baja de peso \\
\hline Otros síntomas & No & No & $\begin{array}{l}\text { Tos seca, decaimiento, dia- } \\
\text { foresis }\end{array}$ & No & Anorexia, depresión \\
\hline Duración & 3 semanas & 1 mes & $\begin{array}{l}3 \text { meses. Síntomas reapare- } \\
\text { cen al año sin tratamiento }\end{array}$ & 9 meses & 1 mes \\
\hline Fiebre & No & No & No & No & No \\
\hline Hematocrito (\%) & 48,8 & 40,1 & 40,4 & 46 & \\
\hline Linfocitos $/ \mathrm{mm}^{3}$ & & 8.000 & 2.450 & 3.210 & \\
\hline VHS mm/h & 1 & 2 & 7 & 10 & \\
\hline ELISA para VIH & Negativo & Negativo & Negativo & Negativo & No solicitado \\
\hline Baciloscopia & Positiva & Positiva & No posible por tos escasa & $\begin{array}{l}3 \text { de } 4 \text { muestras positivas } \\
(+)\end{array}$ & No posible por ausencia tos \\
\hline Baciloscopia en LBA & & & negativa & No aplicable & negativa \\
\hline Cultivo en LBA & & & positivo & No aplicable & positivo \\
\hline Imágenes & & $\begin{array}{l}\text { Ecografía: engrosamiento } \\
\text { del celular subcutáneo, } \\
\text { fístula, adenopatías regio- } \\
\text { nales múltiples }\end{array}$ & $\begin{array}{l}\text { TAC: Infiltrados noduli- } \\
\text { llares, bronquiectasias, } \\
\text { árbol en brote, linfonodos } \\
\text { mediastínicos. Lesiones } \\
\text { progresan luego de un año } \\
\text { sin tratamiento }\end{array}$ & $\begin{array}{l}\text { Radiografía: Infiltrados } \\
\text { intersticiales bilaterales de } \\
\text { predominio en las bases, } \\
\text { sin efusión pleural. No } \\
\text { adenopatías }\end{array}$ & $\begin{array}{l}\text { TAC: Infiltrados noduli- } \\
\text { Ilares bilaterales lóbulos } \\
\text { superiores, árbol en brote, } \\
\text { bronquiectasias, linfonodos } \\
\text { mediastínicos }\end{array}$ \\
\hline Especie bacteriana & M. chelonae & M. fortuitum & $\begin{array}{l}\text { M. intracellulare } \\
30 \text { colonias y } 50 \text { colonias } \\
\text { en un segundo cultivo un } \\
\text { año después }\end{array}$ & $\begin{array}{l}\text { M. intracellulare } \\
\text { Incontables }\end{array}$ & M. kansasii \\
\hline Latencia muestra-informe final & 42 días & & 76 días & 89 días & 40 días \\
\hline Manejo inicial & $\begin{array}{l}\text { Tratamiento } \\
\text { específico }\end{array}$ & $\begin{array}{l}\text { Antibioterapia común y } \\
\text { resección quirúrgica }\end{array}$ & $\begin{array}{l}\text { Tratamiento anti TBC por } \\
\text { aviso de cultivo positivo a } \\
\text { micobacteria }\end{array}$ & $\begin{array}{l}\text { Tratamiento anti TBC por } \\
\text { tinción positiva en expec- } \\
\text { toración }\end{array}$ & Sin tratamiento \\
\hline Evolución & Favorable & Favorable & $\begin{array}{l}\text { Se suspende primer trata- } \\
\text { miento para TBC, evolución } \\
\text { favorable con recaída } \\
\text { sintomática a los } 12 \text { meses } \\
\text { de seguimiento }\end{array}$ & Favorable & $\begin{array}{l}\text { Favorable a } 4 \text { años de } \\
\text { seguimiento }\end{array}$ \\
\hline Clasificación BTS & & & Con enfermedad pulmonar & Con enfermedad pulmonar & Sin enfermedad pulmonar \\
\hline Clasificación ATS 2007 & & & Con enfermedad pulmonar & Con enfermedad pulmonar & Con enfermedad pulmonar \\
\hline
\end{tabular}

que resultó positiva para Mycobacterium fortuitum a los 13 días de incubación en medio sólido. La identificación se efectuó mediante hibridización reversa. Se indicó resección quirúrgica de las adenopatías inguinales más grandes, se suspendió el tratamiento antituberculoso y se indicó triple terapia con amikacina por 21 días asociado a claritromicina y levofloxacina por seis meses. La evolución posterior fue favorable.

\section{Infecciones pulmonares}

Se trató de mujeres post-menopáusicas (casos 2 al 5 en la Tabla 1). Dos tenían antecedente de patología pulmonar previa (asma y EPOC) y una antecedente de TBC esofágica tratada. Ninguna era usuaria de corticosteroides sistémicos o inhalatorios y tampoco reportaron otras infecciones oportunistas remotas o recientes.

Las tres se presentaron con baja de peso y/o tos con 
expectoración pero en uno de ellos se pudo establecer que la baja de peso estaba relacionada a un cuadro depresivo. En dos casos con información disponible no se objetivó anemia, linfopenia ni aumento en la eritrosedimentación. Ningún paciente tuvo fiebre y sólo una de ellas diaforesis. En un caso se contó con radiografía de tórax que mostró infiltrados intersticiales. En los otros dos se realizó una TAC de tórax que demostró la presencia de bronquiectasias, infiltrados nodulillares, árbol en brote y linfonodos mediastínicos (Figura 3). Ningún caso presentó efusión pleural o cavitación. En uno de nuestros pacientes (caso 4), la TAC efectuada un año después del diagnóstico inicial y sin tratamiento específico, demostró progresión de los nodulillos pulmonares (Figura 4).

En estos tres casos se sospechó inicialmente TBC pulmonar por el cuadro clínico y los hallazgos en las imágenes. Las muestras obtenidas para estudio de micobacterias fueron expectoración en un caso y en dos fibrobroncoscopia con lavado-bronquioalveolar (LBA). La baciloscopia de expectoración resultó positiva en dos muestras separadas (caso 5). Las muestras obtenidas por fibrobroncoscopia resultaron negativas con tinción de Ziehl-Neelsen en los dos casos restantes. En dos de los tres casos con aislados pulmonares, se identificó $M$. intracellulare y en uno $M$. kansasii. La latencia entre la toma de la muestra y el informe del cultivo definitivo tuvo un rango de 40 a 89 días.

En dos de los tres casos se contó con una prueba de ELISA para VIH negativa y en el restante este examen no fue solicitado por el equipo tratante por no plantearse la sospecha. El seguimiento hasta junio de 2014 (cuatro años) no ha demostrado condición alguna indicadora de inmunosupresión.

\section{Tratamiento}

En dos de estos tres casos se inició tratamiento para TBC (casos 3 y 4), antes de disponer del informe definitivo. Una de estas pacientes no lo toleró por molestias gastrointestinales y lo abandonó (caso 3), en paralelo a la identificación de una micobacteria atípica en el cultivo. La buena evolución inicial de la paciente post-suspensión del tratamiento hizo desestimar un tratamiento específico para esta micobacteria. Sin embargo, los mismos síntomas iniciales reaparecieron luego de un año, obligando a efectuar una segunda fibrobroncoscopia con LBA. Las imágenes en la TAC de tórax progresaron (Figura 4). Una RCP para M. tuberculosis resultó negativa y el cultivo confirmó la persistencia de $M$. intracellulare. Se inició un tratamiento trisemanal con etambutol, rifampicina y azitromicina que recibió durante aproximadamente tres meses pero suspendió por intolerancia digestiva. El seguimiento a dos años plazo indica que la paciente continua estable con escasa tos y sin bajar de peso.

En el segundo caso, luego de confirmarse el hallazgo

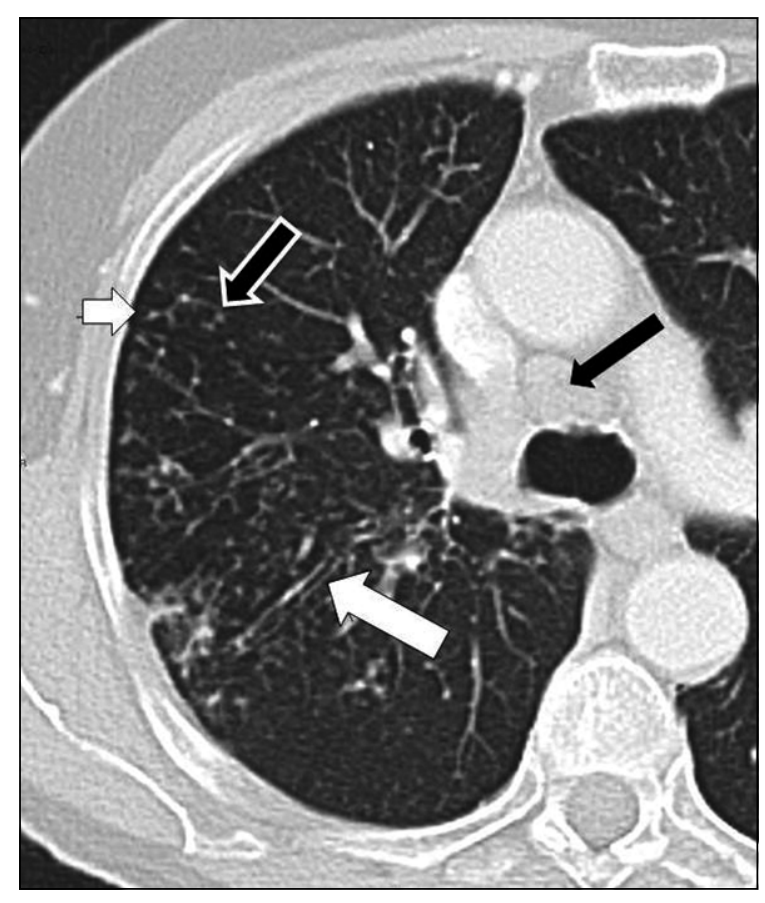

Figura 3. Corte axial de TAC donde se aprecia imágenes nodulillares centro lobulares (flecha negra con borde blanco) y árbol en brote (flecha blanca corta). Se reconocen bronquiectasias (flecha blanca oblicua) y adenopatía precarinal (flecha negra).

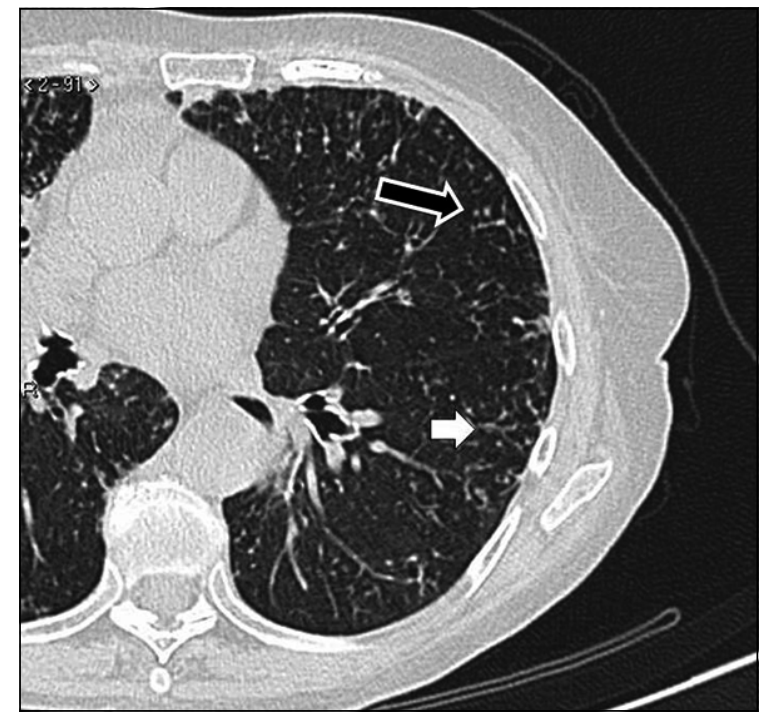

Figura 4. Progresión de imágenes nodulillares (flecha negra con borde blanco) y árbol en brote (flecha blanca corta) al año de seguimiento del caso presentado en la Figura 3 (caso 3).

de $M$. intracellulare el tratamiento para TBC se mantuvo durante la fase diaria y parte de la fase bisemanal (caso 4) por la buena respuesta clínica, pero fue cambiado a un esquema trisemanal con etambutol, rifampicina y azitromicina (500 mg/día) por un año, esquema que completó y permanece desde entonces en buenas condiciones.

Por su parte, en el caso 5 no se inició tratamiento para TBC debido a las buenas condiciones clínicas de la paciente y al hallazgo de una micobacteria atípica en expectoración. 


\section{Discusión}

Los casos relatados ponen de relieve la emergencia de micobacterias atípicas en pacientes sin evidencias de inmunosupresión en nuestro país y las dificultades diagnósticas y terapéuticas asociadas. La concentración de casos en inmunocompetentes en años recientes sin antecedentes de aislados de este tipo en años previos para este tipo de pacientes, sugiere una posible curva en ascenso. Este fenómeno coincide con el aumento de hospitalizaciones por esta causa en Estados Unidos de América en pacientes sin infección por $\mathrm{VIH}^{9}$. Como se comentó previamente, las infecciones por este tipo de micobacterias también han aumentado en el Reino Unido $^{1,2}$. En Argentina, esta emergencia también ha sido descrita en años recientes ${ }^{10}$.

Antes de la pandemia de SIDA, aproximadamente 90\% de las micobacterias atípicas eran aisladas de muestras pulmonares, 3\% desde ganglios, la misma cifra desde piel y tejidos blandos, y menos de $1 \%$ en asociación con formas diseminadas ${ }^{11}$. Con el advenimiento de la pandemia de SIDA, las muestras asociadas a infecciones diseminadas incrementaron su participación pero no llegaron a superar el 5\% del total de muestras identificadas, manteniéndose el predominio de muestras respiratorias, que representa cerca de $75 \%$ de los aislados identificados. De esta manera, el hecho de que tres de nuestros cinco casos estén asociados a muestras respiratorias, subraya la relevancia de este origen y sugiere que en el futuro, a medida que se mantenga la declinación progresiva de la TBC en nuestro país, el valor predictor positivo de los exámenes tradicionales para reconocer cuadros de TBC podría reducirse y generar una confusión clínica con decisiones inapropiadas ya fuese sobre el inicio de la terapia o sobre los compuestos óptimos que deben ser aplicados, tal como sucedió en nuestros pacientes.

Debido a la latencia de los cultivos en medios tradicionales para, primero obtener desarrollo de colonias y posteriormente, efectuar pruebas de identificación a nivel de especie (lo que puede superar los 60 días), se favorece el uso de una terapia innecesaria o inapropiada, la que se puede prolongar por varias semanas antes de efectuar ajustes terapéuticos. Ciertamente, el advenimiento de pruebas moleculares ya presentes en el Laboratorio de Referencia Nacional ${ }^{12}$, permite acelerar el diagnóstico de la especie involucrada, aunque requiere siempre completar una fase de crecimiento en cultivo antes de tener material suficiente para la fase molecular.

Debido a la presencia ubicua de micobacterias atípicas en el ambiente, el hallazgo aislado de una de ellas en muestras naturalmente contaminadas como las de vía aérea, no implica necesariamente enfermedad. Este problema ha sido abordado por al menos dos guías extranjeras que exigen varias muestras positivas junto a alteraciones pulmonares para establecer el diagnóstico de enfermedad pulmonar atribuible y por ende justificar la terapia ${ }^{2,11}$. Dos de nuestros tres casos pulmonares, cumplieron con los criterios de la British Thoracic Society y de la American Thoracic Society publicados el año 1997 para establecer la existencia de enfermedad pulmonar ${ }^{2}$. Sin embargo, de acuerdo a los nuevos criterios de la American Thoracic Society revisados y publicados el año 2007, los tres casos con aislados pulmonares califican para enfermedad pulmonar ya que basta el desarrollo de una micobacteria atípica desde el LBA sin necesidad de obtener dos aislados separados ${ }^{11}$. Uno de nuestros pacientes fue calificado en forma discordante bajo esta premisa y ha permanecido estable por varios años, lo que cuestiona la especificidad de un cultivo único de micobacteria atípica desde el LBA como criterio suficiente.

La naturaleza del diseño de estos criterios, que exigen en general muestras separadas, no acelera la certeza diagnóstica, y en países donde aún existe la TBC como en Chile, no es fácil esperar estos resultados y postergar la decisión de tratamiento. En la actualidad, en ocasiones aún es necesario suspender la terapia para TBC o adecuar el esquema para cubrir micobacterias atípicas. Esto trae tres problemas secundarios: la necesidad de anular la notificación de TBC y suspender la quimioprofilaxis en los contactos, reacciones adversas innecesarias a medicamentos y finalmente, conflictos con el paciente por recibir una terapia innecesaria por varias semanas. Estos problemas ya han sido advertidos en la literatura médica ${ }^{13}$.

La sospecha precoz de una infección pulmonar por micobacterias atípicas podría plantearse ante un paciente sintomático con tinción positiva en expectoración pero con una prueba de liberación de interferon gamma (IGRA) para TBC negativa ${ }^{14}$ y también ante una tinción positiva con una amplificación molecular negativa para $M$. tuberculosis complex (Tabla 2). Sin embargo, la primera condición es discutible porque un paciente con TBC activa puede tener una prueba IGRA negativa. No obstante, estas claves para una sospecha apropiada podrían evitar la demora excesiva en el manejo adecuado de los casos y minimizar errores. El sistema de diagnóstico imperante actualmente en Chile, basado en tinción y cultivos en medios sólidos, y no en la incorporación de medios de cultivos líquidos o métodos de detección molecular rápida, facilita la lentitud diagnóstica y la confusión terapéutica.

En nuestra serie de casos pulmonares fue posible observar un patrón ya descrito en pacientes sin evidencias de inmunosupresión y que incluye la estrecha asociación con mujeres post-menopáusicas, antecedentes de patología pulmonar subyacente e imágenes pulmonares caracterizadas por infiltrados nodulillares con bronquiec$\operatorname{tasias}^{2,11,15,16}$. Es posible, que en el futuro, el aislamiento de micobacterias atipícas en nuestro país provenientes de pacientes sin evidencias de inmunosupresión, mantengan 
esta asociación particular, especialmente al considerar que la mayor parte de las muestras se originan en este parénquima. No observamos en esta pequeña serie, el patrón fibrocavitario pulmonar, el que además de la TBC clásica también ha sido descrito en micobacterias atípicas.

Dos casos de esta serie presentaron infecciones asociadas a piel y tejidos blandos por diferentes especies de crecimiento rápido lo que facilita el diagnóstico precoz y la terapia apropiada ${ }^{11}$. Mycobacterium chelonae es un microorganismo característicamente asociado a infecciones de piel y tejidos blandos tal como sucedió en uno de nuestros casos y Mycobacterium fortuitum puede no sólo causar infección de piel y tejidos blandos sino también infección del sitio quirúrgico y bacteriemia relacionada a catéter, habitualmente por inoculación directa ${ }^{10,17}$. Para M. fortuitum se han descrito infecciones de piel y tejidos blandos graves asociadas a tatuajes y baños en salones de pedicure al igual que para otras micobacterias de crecimiento rápido ${ }^{18,19}$. Estas especies pueden generar una enfermedad grave cuando progresan más allá del compromiso cutáneo, incluyendo tejidos blandos, compromiso óseo, pulmonar o diseminado. Un aspecto interesante a destacar para estas infecciones cutáneas es que el crecimiento de $M$. chelonae se puede dar en medios habituales pero $M$. fortuitum requiere medios especiales a pesar de que ambas pertenecen al grupo de micobacterias de crecimiento rápido.

El tratamiento de los pacientes infectados con micobacterias atípicas, enfrenta a su vez diferentes problemas. El primero de ellos y en contraposición a la experiencia con $\mathrm{TBC}$, es que la susceptibilidad antimicrobiana in vitro no tiene una correlación clara con la respuesta clínica del paciente, existiendo por experiencia o investigación sólo algunas asociaciones establecidas ${ }^{2,20}$. En segundo lugar, la susceptibilidad no es similar a la que presenta $M$. tuberculosis y, por lo tanto, sus esquemas no son en general aplicables sobre las micobacterias atípicas. En tercer lugar, el uso apropiado de terapias siguiendo las recomendaciones establecidas por guías clínicas, tiene un mejor pronóstico que la adopción arbitraria de compuestos a nivel local. Por ejemplo, en el caso de infecciones por $M$. avium o $M$. intracellulare, la tasa de recurrencia o muerte en pacientes sin infección VIH es más del doble al aplicar esquemas locales $(78 \%)$ que al seguir recomendaciones consensuadas $(31 \%)^{2}$. Y en cuarto lugar, los tratamientos son en general más prolongados, superando el año de tera-

Tabla 2. Similitudes y diferencias entre infecciones clínicas por Mycobacterium tuberculosis y micobacterias atípicas

\begin{tabular}{|c|c|c|}
\hline Parámetro & Mycobacterium tuberculosis & Micobacterias atípicas \\
\hline Reservorio & Ser humano & Ambiental \\
\hline Transmisibilidad entre seres humanos & Sí & No \\
\hline Establece infección latente & Sí & No \\
\hline Sitio de localización más frecuente & Pulmonar & Pulmonar \\
\hline Situación epidemiológica en Chile & $\begin{array}{l}\text { En declinación con tasas cercanas a 12/100.000 habi- } \\
\text { tantes }\end{array}$ & $\begin{array}{l}\text { Posiblemente en expansión pero con tasas }<1 / 100.000 \\
\text { habitantes }\end{array}$ \\
\hline $\begin{array}{l}\text { Identificación por tinción o cultivo positivo sin especificar } \\
\text { la especie desde muestra respiratoria }\end{array}$ & Un resultado positivo implica tratamiento & $\begin{array}{l}\text { Un resultado positivo no implica tratamiento ya que se } \\
\text { requieren varias muestras positivas, excepto en LBA o si } \\
\text { tiene imágenes compatibles }\end{array}$ \\
\hline Sospecha microbiológica & & Tinción positiva junto a RPC negativa para $M$. tuberculosis \\
\hline $\begin{array}{l}\text { Identificación por tinción o cultivo positivo sin especificar } \\
\text { la especie desde un sitio estéril }\end{array}$ & Implica tratamiento & Implica tratamiento \\
\hline Enfermedad notificable & Sí & No \\
\hline Prueba de tuberculina-PPD & Puede ser positiva & Puede ser positiva \\
\hline Prueba de liberación gamma-interferon-IGRA & Puede ser positiva & Negativa \\
\hline Tratamiento médico & $\begin{array}{l}\text { Normado, Prolongado, } 3 \text { ó } 4 \text { fármacos, esquemas acor- } \\
\text { tados disponibles por } 6 \text { meses }\end{array}$ & $\begin{array}{l}\text { No normado. Según especie, incluye } 1 \text { a } 3 \text { fármacos y } \\
\text { puede variar entre } 2 \text { meses y en otros casos superar los } \\
18 \text { meses }\end{array}$ \\
\hline Gratuidad-Cobertura del tratamiento en Chile & Sí & Si en caso de fármacos similares al tratamiento de la TBC \\
\hline Tratamiento quirúrgico & Rara vez necesario & Necesario en algunas localizaciones y según especie \\
\hline Quimioprofilaxis de contactos & Sí & No \\
\hline
\end{tabular}


pia. Afortunadamente, desde el año 2013 existe cobertura gratuita en Chile para el tratamiento de estas micobacterias a través del Programa de Control y Eliminación de la TBC aunque sin incluir los macrólidos. En el caso de $M$. intracellulare en pacientes con enfermedad pulmonar, la recomendación es aplicar como compuesto fundamental azitromicina o claritromicina (Tabla 2) junto a etambutol y rifampicina hasta lograr 12 meses con cultivos negativos. En el caso de infecciones sin cavitaciones se pueden aplicar estos compuestos en forma trisemanal, lo que no es posible en caso de haber cavitaciones. Por su parte, para infecciones pulmonares por M. kansasii, la condición de mayor similitud con $M$. tuberculosis, la recomendación es usar isoniazida, rifampicina y etambutol al menos 18 meses y que incluyan 12 meses de cultivos negativos.

Para el tratamiento de infecciones cutáneas por $M$. chelonae sólo es necesario claritromicina. Su duración no ha sido claramente establecida pero una experiencia nacional utilizó este tratamiento por dos meses con buenos resultados ${ }^{7}$. En caso de infección mayor, se sugieren tratamientos por al menos cuatro meses ${ }^{11}$.

El tratamiento recomendado para las infecciones por M. fortuitum limitadas a piel y tejidos blandos es habitualmente oral con dos antimicrobianos asociados, pudiendo ser las opciones cotrimoxazol, claritromicina, doxiciclina o levofloxacina. Las formas graves requieren de una fase inicial parenteral por dos a seis semanas, con al menos dos compuestos activos, siendo los más comúnmente usados amikacina, imipenem, tobramicina y cefoxitina con una duración total de 6 a a12 meses. La cirugía está indicada para enfermedad cutánea muy extensa, formación de abscesos o pobre respuesta a tratamiento oral. El caso incluido en esta serie requirió por su extensión un tratamiento combinado médico-quirúrgico y un tratamiento asociado prolongado.

El aislamiento de micobacterias atípicas cuestiona el paradigma actual de enfoque de pacientes en los que se recibe una muestra con bacilos ácido-alcohol resistentes o un cultivo positivo inicial sin identificación de especie (Mycobacterium spp), especialmente si se considera que podría corresponder a una especie emergente. En la Tabla 2 se mencionan algunos contrastes y similitudes entre los dos grupos de micobacterias.

Esta serie clínica tiene limitaciones relacionadas al pequeño número de casos que incluye, lo que impide establecer un perfil propio, y por ello, los aspectos señalados deben asumirse como un comentario clínico que requiere un análisis en series mayores. De la misma manera, la prueba serológica para infección por VIH no fue realizada en uno de nuestros casos y es posible por lo tanto, que este paciente hayan tenido SIDA. Sin embargo, esta probabilidad es remota debido a que las infecciones por micobacterias atípicas en pacientes infectados con VIH se dan en fases de inmunosupresión avanzada con otros eventos oportunistas previos, característica no presente en el caso comentado. Además, el seguimiento clínico a cuatro años de plazo no ha dado señales de tal posibilidad.

En conclusión, esta pequeña serie de casos de pacientes afectados por micobacterias atípicas sin evidencia de inmunosupresión asociada, revela la emergencia de estas especies en nuestro país, la estrecha asociación de las muestras de origen pulmonar con mujeres postmenopáusicas con patología pulmonar subyacente, la aparición ocasional de ellas en muestras clínicas de otro origen y las dificultades diagnósticas y terapéuticas generadas por su hallazgo.

\section{Resumen}

El objetivo de este trabajo es reportar la experiencia acumulada sobre infecciones por micobacterias atípicas en pacientes sin inmunosupresión. Entre el año 2008 y 2013 se observaron cinco pacientes con infección por micobacterias atípicas: dos con infección cutánea y tres con infección pulmonar. Ninguno de estos pacientes tenía evidencias de inmunosupresión. Un paciente con bursitis de codo por $M$. chelonae tuvo un estudio citoquímico con aumento de celularidad de predominio mononuclear y desarrollo de bacterias al quinto día; respondió favorablemente a claritromicina. Un caso con infección cutánea por $M$. fortuitum evolucionó en forma prolongada con supuración ganglionar antes del diagnóstico y el cultivo solicitado a los 13 días fue positivo. Los tres pacientes con aislados pulmonares presentaron tos y expectoración y tenían en común ser mujeres en edad post-menopáusica y presentar pequeños infiltrados nodulares asociados a bronquiectasias en el estudio de imágenes pulmonares, un patrón descrito en la literatura científica. En estos tres casos, la latencia entre la toma de muestra y el informe definitivo tuvo un rango de 40 a 89 días. El aislamiento de micobacterias atípicas en muestras de expectoración en pacientes sin inmunosupresión se da en un contexto típico pero plantea dificultades diagnósticas y terapéuticas. El lento crecimiento de estos microorganismos en el laboratorio contribuye a este problema. 


\section{Referencias bibliográficas}

1.- Moore J E, Kruijshaar M E, Ormerod L P, Drobniewski F, Abubakar I. Increasing reports of non-tuberculous mycobacteria in England, Wales, and Northern Ireland, 1995-2006. BMC Public Health 2010: 10: 612.

2.- Henry M T, Inamdar L, O'Riordain D, Schweiger M, Watson J P. Nontuberculous mycobacteria in non-HIV patients: epidemiology, treatment, and response. Eur Respir J 2004; 23: 741-46.

3.- Scappaticcio A, Velasco M, Leiva T, Rodríguez J C. Frecuencia de micobacterias ambientales en Chile en el 2008. Rev Chil Enf Respir 2011; 27: 214-2.

4.- Strickler A, Boza M L, González B, Márquez G, Bustamante J. Infección diseminada por BCG en la Región de Los Lagos, Chile: Reporte de 5 casos clínicos. Rev Chil Enf Respir 2009; 25: 29-38.

5.- Chernilo S, Trujillo S, Kahn M, Paredes M, Echevarría G, Sepúlveda C. Enfermedades pulmonares en pacientes infectados con VIH hospitalizados en el Instituto Nacional del Tórax. Rev Med Chile 2005; 133: 517-24.

6.- Jensen W, Cardemil F. Infección diseminada por Mycobacterium intracellulare en paciente VIH: reporte de un caso y revisión de la literatura. Bol Hosp Viña del Mar 2009; 65 (3/4): 123-9.

7.- Pérez C M, Guzmán A M, García P, Rabagliati R, Labarca J, Turcios R, et al. Outbreak of rapidly-growing mycobacterial skin abscesses following cosmetic injections in Chile. 41th Annual Meeting of the Infectious Diseases Society of America, San Diego, USA. October 2003.

8.- Saldías F, Tirapegui F, Díaz O. Infección pulmonar por Mycobacterium avium complex en el huésped inmunocompetente Rev Chil Enf Respir 2013; 29: 162-7.

9.- Billinger M E, Olivier K N, Viboud C, Montes de Oca R, Steiner C, Holland S M, et al. Nontuberculous mycobacteria-associated lung disease in hospitalized persons, United States, 1998-2005. Emerg Infect Dis 2009; 15 : 1562-9.

10.- Gutiérrez M E, Villar H E, Montoto M, Vicente A, Longo L M, Hoffman N. Micobacteriosis pulmonar en pacientes HIV negativos en la ciudad de Buenos Aires, años 2003-2004. Medicina (Buenos Aires) 2006; 66 : $139-43$.

11.- Griffith D E, Aksamit T, Brown-Elliot B A, Catanzaro A, Daley C, Gordin F, et al. An official ATS/IDSA statement: Diagnosis, treatment, and prevention of nontuberculous mycobacterial diseases. Am J Respir Crit Care Med 2007; 175: 367-416.

12.- Leiva T. Evaluación de una técnica de hibridación reversa para identificación rápida de micobacterias en Chile. Rev Chil Enf Respir 2012; 28: 9-15.

13.- Van Crevel R, de Lange W C M, Vanderpuye N A, van Soolingen D, Hoogkamp-Korstanje J A A, van Deuren M, et al. The impact of nontuberculous mycobacteria on management of presumed pulmonary tuberculosis. Infection 2001; 29 : 59-63.
14.- Han Y M, Kim H S, Kim C H, Kang H J, Lee K M. Analysis of patients with positive acid-fast bacilli culture and negative T-SPOT. TB results. Korean J Lab Med 2010; 30: 414-9.

15.- Dailloux M, Abalain M L, Laurain C, Lebrun L, Loos-Ayav C, Lozniewski A, et al. Respiratory infections associated with nontuberculous mycobacteria in non-HIV patients. Eur Respir J 2006; 28: 1211-5.

16.- Bodle E E, Cunningham J A, Della-Latta P, Schluger N W, Saiman L. Epidemiology of nontuberculous mycobacteria in patients without HIV infection, New York City. Emerg Infect Dis 2008; 14: 390-6.

17.- Wallace R J Jr, Swenson J M, Silcox V A, Good R C, Tschen J A, Stone M S. Spectrum of disease due to rapidly growing mycobacteria. Rev Infect Dis 1983; 5: 657-79.

18.- Stout J E, Gadkowski L B, Rath S, Alspaugh J A, Miller M B, Cox G M. Pedicure-associated rapidly growing mycobacterial infection: an endemic disease. Clin Infect Dis 2011; 53: 787-92.

19.- Falsey R R, Kinzer M H, Hurst $S$, Kalus A, Pottinger P S, Duchin J S, et al. Cutaneous inoculation of nontuberculous mycobacteria during professional tattooing: a case series and epidemiologic study. Clin Infect Dis 2013; 57: e143-7.

20.- Heginbothom M L. The relationship between the in vitro drug susceptibility of opportunistic mycobacteria and their in vivo response to treatment. Int J Tuberc Lung Dis 2001; 5: 539-45. 